\title{
Chronik der Medienentwicklung in Deutschland 2002
}

\author{
Christiane Matzen
}

1. Medienregulierung (Medienpolitik / Medienrecht)

2. Medienunternehmen

3. Medienmärkte

3.1 Medienangebote /Medieninhalte Presse

3.2 Medienangebote / Medieninhalte Rundfunk

3.3 Werbung / Marktanteile

4. Mediennutzung

\section{Medienregulierung (Medienpolitik / Medienrecht)}

Der 13. Bericht der Kommission zur Ermittlung des Finanzbedarfs der Rundfunkanstalten (KEF), der am 23. Januar in Berlin vorgestellt wird, kommt zu dem Ergebnis, dass für die laufende Gebührenperiode mit einem Überschuss bei der ARD und beim DeutschlandRadio (DLR), hingegen mit einem Defizit beim ZDF zu rechnen ist. Die derzeitige Rundfunkgebühr von monatlich 16,15 Euro sei folglich bis Ende 2004 ausreichend. Darüber hinaus spricht sich die Kommission für eine weitgehend politisch vorzugebende strengere Definition der Online-Aktivitäten der öffentlich-rechtlichen Veranstalter und damit für eine Begrenzung der diesbezüglichen Aufgaben aus. ${ }^{1}$ Wie die Frage der Gebühr für internetfähige Computer müsse die Frage der wünschenswerten und auftragsgebundenen Online-Aktivitäten politisch entschieden werden, bevor sich die KEF damit befasse.

Der Bundestag verabschiedet am 25. Januar in zweiter und dritter Lesung das Urhebervertragsgesetz. Das Gesetz stärkt die vertragliche Stellung von Urhebern und ausübenden Künstlern.

Am 26. Februar untersagt das Bundeskartellamt dem amerikanischen Investor Liberty Media den Kauf von sechs Fernsehkabelnetzen der Telekom-Tochter Kabel Deutschland GmbH. Der Zusammenschluss führe zur Verstärkung marktbeherrschender Stellungen sowohl auf dem Markt für die Belieferung von Endkunden mit Rundfunksignalen (Endkundenmarkt Kabelfernsehen) als auch dem Markt für die Einspeisung von Signalen in Breitbandkabelnetzen (Einspeisemarkt) und dem Markt für die Belieferung der Netzbetreiber der Netzebene 4 mit Signalen der Netzebene 3 (Signallieferungsmarkt). Bei einem Erwerb der Breitbandkabelnetze durch Liberty stünden die Verbraucher schlechter da, als wenn die Deutsche Telekom vorerst Eigentümer des Netzes bliebe.

Das Saarland gibt sich mit seinem neuen Mediengesetz vom 27. Februar ${ }^{2}$ einen gemeinsamen Regelungsrahmen für Rundfunk (Radio/Fernsehen) und Presse.

1 Die Überlegungen der KEF im Hinblick auf Konzepte und Vorgaben bezüglich der OnlineAktivitäten von ARD, ZDF und DeutschlandRadio sind dokumentiert in epd medien 6/2002, S. $28 \mathrm{f}$.

2 Dokumentiert in Funkkorrespondenz 13-14/2002, Beilage. 
Mitte März verabschiedet die Bundesregierung einen Bericht zur „Informationsgesellschaft Deutschland“3, die sich „,in den letzten Jahren eindrucksvoll entwickelt und eine europaweite Spitzenposition erreicht hat“. Der Bericht bilanziert die Umsetzung des 1999 von der Bundesregierung initiierten Aktionsprogramms „Innovation und Arbeitsplätze in der Informationsgesellschaft des 21. Jahrhunderts“. Unter anderem wird als Erfolg genannt, dass es zu Beginn des Ausbildungsjahres 2001 über 70.000 Ausbildungsstellen in IT- und Medienberufen und über 10.000 Green-Card-Inhaber gegeben habe. Die Branche für Informations- und Kommunikationstechnologien beschäftigt dem Bericht zufolge über 800.000 Menschen; der durch E-Commerce erzielte Umsatz hat in Deutschland im Jahr 2001 bei 20 Mrd. Euro gelegen.

Das aus sieben Paragrafen bestehende neue Zugangskontrolldiensteschutz-Gesetz $(\mathrm{ZKDSG})^{4}$ tritt am 23. März in Kraft. Es soll u. a. Pay-TV-Veranstalter vor Hackern und Decoder-Saboteuren schützen und setzt eine EU-Richtlinie von 1988 um.

Am 4. April veröffentlicht die Regulierungsbehörde für Telekommunikation und Post (RegTP) „Eckpunkte für die Einführung des digitalen Fernsehens“. Darin geregelt wird das Verfahren, mit dem Frequenzen für DVB-T zugeteilt werden. In der Praxis werden die Bundesländer nun bei der Regulierungsbehörde ihren Bedarf an DVB-TFrequenzen anmelden; die Bundesbehörde wird daraufhin diese Frequenzen in der Regel einzeln ausschreiben. Die Landesmedienanstalten hatten sich zuvor mit der RegTP auf dieses Verfahren geeinigt.

Nach dem Amoklauf eines Schülers an einem Erfurter Gymnasium, bei dem 17 Menschen sterben, schlägt Bundeskanzler Gerhard Schröder am 2. Mai nach einem Treffen mit Intendanten öffentlich-rechtlicher und privater TV-Anstalten in Berlin vor, einen Runden Tisch zur Diskussion der Rolle von Gewaltdarstellungen im Fernsehen einzuberufen. Als Teilnehmer an dem Runden Tisch sollen Vertreter der Bundesregierung, der Landesregierungen, die Vorsitzenden der Rundfunkkommission und der Ministerpräsidentenkonferenz sowie Vertreter der öffentlich-rechtlichen und privaten Rundfunkveranstalter, der Internet-Provider sowie der Produzenten von Video- und Computerspielen geladen werden. Der Runde Tisch soll Vorschläge machen, wie der Jugendschutz und die Kontrolle von Gewaltdarstellungen verbessert werden können. Die Arbeitsgemeinschaft der Landesmedienanstalten (ALM) verabschiedet am 27./28. Mai ein Positionspapier „Gewalt in der Gesellschaft - Gewalt in den Medien“5, das sich für die Förderung von Medienkompetenz und für die Erforschung des komplexen Zusammenhangs von gesellschaftlicher Gewalt und Mediengewalt ausspricht. Die Einrichtungen der Selbstkontrolle müssten gestärkt und in die Durchsetzung des Jugendschutzes eingebunden werden.

EU-Wettbewerbskommissar Mario Monti teilt am 3. Juni mit, dass die zentrale Vermarktung der Live-Übertragungsrechte an der Champions League durch den europäischen Fußballverband UEFA zwar erhalten bleiben solle, es werde jedoch einen Kompromiss geben. Dieser sieht vor, dass die UEFA künftig die Medienrechte (TV, Radio, Internet und UMTS) aufteilen und entweder selbst oder aber zusammen mit den Verei-

3 Eine Zusammenfassung des „Fortschrittsberichts der Bundesregierung zum Aktionsprogramm ,Innovation und Arbeitsplätze in der Informationsgesellschaft des 21. Jahrhunderts “" findet sich unter http://www.bundesregierung.de/Themen-A-Z/Wirtschaft-,9170/Informationsgesellschaft-Deuts.htm.

4 Dokumentiert in epd medien 28/2002, S. 23.

5 Dokumentiert in epd medien 43/2002, S. $20 \mathrm{ff}$. 
nen für einen Zeitraum von nur noch 3 Jahren vermarkten wird. Die EU-Kommission hatte die zentrale Vermarktung im Paket für bis zu 4 Jahre im Juli $2001 \mathrm{u}$. a. deshalb beanstandet, weil sie den Wettbewerb zwischen Fernsehsendern verzerre, wenn nur große Medienkonzerne die Rechte finanzieren könnten, und bei der Zentralvermarktung in Paketen immer einige Rechte an Live-Übertragungen nicht genutzt würden, an denen jedoch regionale oder Pay-TV-Sender Interesse gehabt hätten.

Staatsminister Julian Nida-Rümelin, der Kultur- und Medienbeauftragte der Bundesregierung, legt am 5. Juni sein Reformkonzept für den Auslandsrundfunk Deutsche Welle (DW) ${ }^{6}$ vor. Danach sollen die Aufgaben und Zielgruppen der DW neu formuliert, die finanzielle Grundausstattung gesichert und auch neue Kooperationsformen mit anderen Auslandssendern erprobt werden. Neben Angeboten in Radio und Fernsehen sollen auch Mediendienste in den gesetzlichen Auftrag aufgenommen werden. Die weitere Präzisierung des Programmauftrags soll im Rahmen eines strukturierten Selbstregulierungsprozesses zwischen der DW, Bundestag und Bundesregierung geschehen.

Die Bremische Bürgerschaft beschließt am 13. Juni Novellen zum Radio-Bremen-Gesetz und zum Landesmediengesetz, die zum 1. Juli in Kraft treten ${ }^{7}$. Bei Radio Bremen ändern sich dadurch die Zusammensetzung des Rundfunkrats und die Regelungen zum Mitbestimmungsrecht sowie der Ausstrahlung von Wahlwerbung. Das Landesmediengesetz enthält neue Regelungen zur Einführung des digitalen terrestrischen Fernsehens und zur Schaffung eines privaten Ballungsraumfernsehens in Bremen.

Der Deutsche Bundestag verabschiedet am 14. Juni das neue Jugendschutzgesetz $(\mathrm{JuSchG})^{8}$, der Bundesrat stimmt am 21. Juni zu. In Kraft treten kann das Gesetz jedoch erst, wenn auch die Länder ihren Teil der Reform abgeschlossen haben. Dies erfolgt am 8. August mit der Verabschiedung des Jugendmedienschutz-Staatsvertrags (JMSTV) ${ }^{9}$ durch die Chefs der Staats- und Senatskanzleien der Bundesländer. Nach Unterzeichnung durch die Ministerpräsidenten und der Ratifizierung durch die Landtage soll der „Staatsvertrag über den Schutz der Menschenwürde und den Jugendschutz in Rundfunk und Telemedien“ am 1. April 2003 in Kraft treten, zusammen mit dem Jugendschutzgesetz des Bundes. Mit dem Jugendmedienschutz-Staatsvertrag errichten die Länder eine zentrale neue Aufsichtsstelle, die Kommission für Jugendmedienschutz (KJM), die abschließend über Fragen des Jugendschutzes für länderübergreifende Angebote entscheidet und dabei - vergleichbar der KEK - als Organ der jeweils zuständigen Landesmedienanstalt fungiert. Sie wird allerdings in vielen Fällen nur dann tätig, wenn die im JMSTV gestärkte Selbstkontrolle versagt. Während der Bund in der Regel für Presse, Videos, CD-ROM, DVD und andere Offline-Medien zuständig ist, umfasst der Kompetenzbereich der Länder Fernsehen, Hörfunk, Internet und andere Online- „Telemedien“. Der Begriff „Telemedien“ steht für Mediendienste und für Teledienste; er soll die bisherigen schwierigen Differenzierungen zwischen beiden Varianten überflüssig machen. Das neue Jugendschutzgesetz des Bundes enthält etliche Vorschriften zum Medienbereich: U. a. wird dort die Tätigkeit der Bundesprüfstelle für jugendgefährdende Medien (bislang „Schriften“) geregelt. Das Jugendschutzgesetz ist insofern ein völlig neues Bundesgesetz, als es zwei Gesetze zusammenführt: das „Gesetz über die Verbrei-

6 Dokumentiert in epd medien 57/2002, S. $17 \mathrm{ff}$.

7 Beide sind veröffentlicht im Gesetzesblatt der Freien Hansestadt Bremen Nr. 26 vom 24. Juli 2002, S. $210 \mathrm{ff}$.

8 Dokumentiert in epd medien 57/2002, S. 3 ff.

9 Dokumentiert in epd medien 72/2002, S. 3 ff. 
tung jugendgefährdender Schriften und Medieninhalte“ (GjS) und das „Gesetz zum Schutze der Jugend in der Öffentlichkeit" (JÖSchG).

Die Fusion des Sender Freies Berlin (SFB) und des Ostdeutschen Rundfunk Brandenburg (ORB) wird am 25. Juni durch die Unterzeichnung eines „Staatsvertrags über die Errichtung einer gemeinsamen Rundfunkanstalt der Länder Berlin und Brandenburg “10 beschlossen. Nach Ratifizierung durch die Landesparlamente tritt der Vertrag am 1. Dezember in Kraft, am 18. Dezember konstituiert sich der 30-köpfige Rundfunkrat. Die neue Anstalt Rundfunk Berlin Brandenburg (RBB) mit Doppelsitz in Berlin und Potsdam soll den SFB und ORB ablösen und zum 30. Juni 2003 ihre Arbeit aufnehmen.

Am 1. Juli tritt die 6. Novelle des Rundfunkstaatsvertrags in Kraft und damit auch neue Medienkonzentrationsregeln, die insbesondere die verwandten Märkte (etwa Presse) mit in die Berechnung einbeziehen. Zudem wurde der Mediendienste-Staatsvertrag an die seit Juni 2000 gültige EU-Richtlinie zum elektronischen Geschäftsverkehr (ECommerce) angepasst; dies betrifft vor allem die Haftungsregeln.

Im Rahmen eines Zwischenberichts stellt Staatsminister Julian Nida-Rümelin am 4. Juli die ersten Umsetzungsschritte sowie die Grundlinien der im Jahr 2003 anstehenden Novellierung des Filmförderungsgesetzes vor. Bereits erreicht seien eine massive Erhöhung des Etats, die Einführung neuer Filmpreise (z. B. ein Filmpreis in Gold für den besten Kinderspielfilm) sowie Lockerungen der Zweckbindungen für die Preise. Im Zuge der Reform des Filmförderungsgesetzes sollen in einem nächsten Schritt die Fördermaßnahmen des Bundes grundlegend modifiziert und bessere Rahmenbedingungen für die deutsche Produzentenlandschaft geschaffen werden. Auf der Anhörung vor dem Bundestagsausschuss für Kultur und Medien zur Filmpolitik und zur anstehenden Novellierung des Filmförderungsgesetzes am 13. März hatte die Filmstiftung NordrheinWestfalen für eine klarere Aufgabenverteilung zwischen Bund und Ländern plädiert.

Die Medienanstalt Berlin-Brandenburg beschließt im Juli, als Pilotprojekt in Berlin bis Sommer 2003 die terrestrische analoge Rundfunkübertragung komplett zugunsten der digitalen Technik abzuschalten. Von der Umstellung betroffen wären rund 140.000 Haushalte (knapp 7 \% der Gebührenzahler), die sich einen Decoder zum Empfang anschaffen müssten. Ungeklärt bleibt, wer die anfallenden Kosten für die gebührenbefreiten Haushalte übernimmt.

Die Landesregierung Nordrhein-Westfalen gibt am 26. Juli bekannt, das Filmbüro NRW ab 2003 nicht mehr als eigenständige Einrichtung fortzuführen. Der Etat für Zwecke kultureller Filmförderung von bislang rund 1,8 Mio. Euro wurde im Landeshaushalt nicht mehr berücksichtigt. Ersatzweise strebt das Kabinett von Wolfgang Clement (SPD) an, Aufgaben und Mitarbeiter des Oberhausener Filmbüros der Filmstiftung NRW in Düsseldorf einzuordnen. Angesichts der Sparzwänge des Landes mit Kürzungen in allen möglichen Bereichen sei es „nicht mehr vermittelbar“, die Filmförderung von Abstrichen verschont zu lassen.

Am 31. Juli tritt in Nordrhein-Westfalen ein neues Landesmediengesetz ${ }^{11}$ in Kraft. Die bisherige Aufsichtsbehörde Landesanstalt für Rundfunk trägt danach den neuen Namen Landesanstalt für Medien Nordrhein-Westfalen (LfM). Sie erhält mit einem 5-köpfigen, vom NRW-Landtag gewählten Medienrat ein neues Organ, das einmal jährlich einen Bericht über Stand und Entwicklung des Rundfunks in NRW erstellt, und

10 Der Staatsvertrag in der Fassung vom 25. Juni 2002 ist dokumentiert in Funkkorrespondenz 27/2002, Beilage, sowie in epd medien 45/2002, S. $23 \mathrm{ff}$.

11 Dokumentiert in Funkkorrespondenz 34/2002, Beilage, sowie in epd medien 63/2002, Beilage. 
statt der bisherigen Rundfunkkommission mit 45 Mitgliedern eine 23-köpfige Medienkommission. Außerdem wird auch der Aufgabenbereich erweitert: Die LfM fördert Medienkompetenz und Aus- und Fortbildung in Medienberufen, genehmigt und kontrolliert private Rundfunkveranstalter, betreibt Forschung und vergibt Qualitätskennzeichen. Entgegen früheren Plänen ist im neuen Gesetz für Zeitungsverlage keine pauschale Beteiligungsgrenze beim Ballungsraumfernsehen mehr vorgesehen.

Für eine Quote für deutschsprachige Musiktitel spricht sich Kultur- und Medienstaatsminister Nida-Rümelin am 16. August auf der Musikmesse „PopKomm“ aus. Hintergrund ist eine von ihm in Auftrag gegebene Studie von Media Control, derzufolge deutsche Musik in einem Großteil der Radios nur einen äußerst geringen Anteil habe. Über die Zielsetzungen einer Quote müssten sich jedoch die für die Rundfunkpolitik zuständigen Bundesländer verständigen.

Das Bundesverfassungsgericht lehnt am 30. August die Annahme einer Verfassungsbeschwerde der FDP ab. Die Liberalen hatten erreichen wollen, dass ihr Kanzlerkandidat Guido Westerwelle an dem Fernsehduell zwischen Bundeskanzler Gerhard Schröder und Kanzlerkandidat Edmund Stoiber bei ARD und ZDF teilnehmen darf. ${ }^{12}$ Es gebe keine „realistische Aussicht“ für den FDP-Kandidaten, das Amt des Bundeskanzlers am 22. September zu übernehmen, ein Recht auf Teilnahme am Fernsehduell scheide daher aus, so das Bundesverfassungsgericht. Zuvor hatten die Liberalen bereits vor dem Verwaltungsgericht und dem Oberverwaltungsgericht Niederlagen erlitten.

Das Europäische Gericht (EuG) in Luxemburg entscheidet am 8. Oktober, dass europäische Privatsender einen besseren Zugang zu den Fernsehsportrechten der Europäischen Rundfunk-Union (EBU) erhalten müssten. Vier Privatsender aus Spanien, Frankreich und Portugal hatten gegen eine Entscheidung der EU-Kommission Klage ${ }^{13}$ eingereicht, da ihnen durch den Sonderstatus der EBU Wettbewerbsnachteile entstünden. 1993 war der EBU gestattet worden, für ihre derzeit 71 Mitglieder, öffentlich-rechtliche Rundfunkanstalten in Europa, Sportrechte zu erwerben. Sie muss aber auch privaten Veranstaltern Zugang durch Unterlizenzregelungen gewähren; diesen Zugang sahen die klagenden Sender nicht gewährleistet.

Im Koalitionsvertrag, den Bündnis 90/Die Grünen und die SPD am 17. Oktober unterzeichnen, wird explizit als Vorhaben formuliert, dass das Deutsche Welle-Gesetz und das Filmförderungsgesetz innerhalb der nächsten vier Jahre novelliert werden sollen. ${ }^{14}$ Die Reform der Medien- und Kommunikationsordnung solle fortgesetzt und Gewaltdarstellungen in allen Medien durch Instrumente der „regulierten Selbstregulierung“ reduziert werden.

Die Ministerpräsidenten beschließen auf ihrer Jahreskonferenz vom 23. bis 25. Oktober, eine Reform der Gebührenfinanzierung des öffentlich-rechtlichen Rundfunks vorzubereiten, die am 1. Januar 2005 in einem neuen Staatsvertrag in Kraft treten soll. Geplant ist, die Gebühren für Radio, TV und Computer künftig pro Haushalt pauschal zu erheben. Für Computer, mit denen über das Internet Rundfunkprogramme empfangbar sind, ist durch ein Moratorium bis Ende 2004 keine Gebühr zu entrichten.

In Baden-Württemberg verabschiedet die Landesregierung am 19. November die Novelle des Landesmediengesetzes Baden-Württemberg, das nach Zustimmung des Landtags zum 1. Februar 2003 in Kraft treten soll. Unternehmen, an denen Parteien unmit-

12 Az.: 2 BvR 1332/02, dokumentiert in epd medien 69/2002, S. 20.

13 Az.: T-185/00, T-216/00, T-299/00 und T-300/00.

14 Die medienrelevanten Passagen sind dokumentiert in epd medien 83/2002, S. $34 \mathrm{ff}$. 
telbar oder mittelbar beteiligt sind, erhalten nach dem neuen Gesetz keine Lizenzen mehr. Außerdem wird die Kabeleinspeisung eines landesweiten privaten Fernsehsenders gesichert und dem Südwestrundfunk (SWR) gestattet, sein digitales Hörfunkprogramm Das Ding künftig auch analog im Kabel zu verbreiten.

Das europäische Parlament verabschiedet am 20. November eine fraktionsübergreifende Entschließung, derzufolge die EU-Kommission bis Ende 2003 ein Grünbuch über den Pluralismus in den Medien und über den Stand neuer Kommunikationstechnologien in den Mitgliedsstaaten Europas und den Beitrittskandidaten zu erstellen hat. Das Grünbuch soll nach Anhörung aller Beteiligten den Sach- und Diskussionsstand zusammenfassen und somit ein Weißbuch oder eine Richtlinie vorbereiten.

\section{Medienunternehmen}

Die Bertelsmann AG gibt am 19. März bekannt, die RTL Group entgegen anders lautender vorheriger Pläne nun doch nicht komplett übernehmen und die freien Aktionäre auszahlen zu wollen. Bertelsmann hält ca. 90 Prozent an der RTL Group, nachdem es 2001 den 22-Prozent-Anteil vom britischen Unternehmen Pearson an RTL übernommen hatte.

Am 9. März wird Markus Schächter zum neuen ZDF-Intendanten gewählt. Er wird am 15. März Nachfolger von Dieter Stolte, der 20 Jahre Intendant des ZDF war. Beim europäischen Kulturfernsehens Arte wird am 4. September der bisherige Vizepräsident Jérôme Clément zum neuen Präsidenten und Nachfolger von Jobst Plog gewählt. Die neue Amtszeit beginnt am 1. Januar 2003 und dauert vier Jahre. Clément war bereits 1991 bis 1998 Präsident von Arte. Helmut Reize wird am 18. Oktober vom 29-köpfigen Rundfunkrat des Hessischen Rundfunks (HR) zum Intendanten gewählt. Sein neues Amt wird er im Mai 2003 als Nachfolger von Klaus Berg antreten, der dann in den Ruhestand geht. Jobst Plog, der am 14. Juni einstimmig für weitere sechs Jahre in seinem Amt als Intendant des Norddeutschen Rundfunks (NDR) bestätigt wurde, wird außerdem ab Januar 2003 ARD-Vorsitzender, nachdem der NDR am 26. November mit der ARD-Geschäftsführung betraut wurde.

Am 8. April beantragt die sämtliche Free-TV-Geschäfte des Medienimperiums von Leo Kirch umfassende Kirch Media AG die Eröffnung eines Insolvenzverfahrens beim Amtsgericht München. Mit dem Zusammenbruch der Kirch Media beginnt die größte Pleite der Wirtschaftsgeschichte in der Bundesrepublik Deutschland. Am 8. Mai folgt die Kirch Pay-TV mit ihren Tochterfirmen Pay-TV Rechtehandel GmbH \& Co. KG, Premiere Beteiligungs $\mathrm{GmbH}$ sowie Beta Digital Gesellschaft für digitale Fernsehdienste mbH. Am 12. Juni meldet auch Leo Kirchs Dachgesellschaft Taurus Holding, die 73 Prozent an der Kirch Media AG und 70 Prozent an Kirch Pay-TV hält, Zahlungsunfähigkeit an. Damit bricht die letzte der drei Hauptsäulen des Firmen-Imperiums zusammen.

In der Folge wird viel spekuliert über mögliche Interessenten für eine Übernahme der Kirch-Unternehmen. Nachdem zunächst vermutet wird, dass sich die Medientycoone Rupert Murdoch und Silvio Berlusconi für Teile des Kirch-Imperiums interessieren könnten, wird ab August von sieben Übernahme-Interessenten berichtet, von denen bald nach Angaben der Insolvenz-Verwalter nur noch drei Bietergruppen in der engeren Wahl bleiben: ein Konsortium aus Commerzbank und dem Hollywood-Studio Columbia Tristar, der amerikanische Medienunternehmer Haim Saban und der französische Medienkonzern TF1 sowie eine Gruppe um die Investmentbank Lehman Brothers. Im Juni wird im Zuge der Suche nach einem Käufer für Kirchs 40\%-Anteil am Axel- 
Springer-Verlag über den Einstieg der finanzkräftigen Essener WAZ-Verlagsgruppe spekuliert. Da es sich bei um vinkulierte Aktien handelt, kann Mehrheitsaktionärin Friede Springer den Verkauf an die WAZ-Gruppe unterbinden. Verhandlungen werden daraufhin - im Sinne der Mehrheitsaktionärin - mit dem Schweizer Verlagshaus Ringier aufgenommen, verkauft wird das Aktienpaket am 8. Oktober allerdings an die Deutsche Bank für 667,3 Mio. Euro.

Ende Oktober scheint dem Verkauf zentraler Unternehmensteile (ProSieben/Sat.1 Media AG und die Filmrechte) der Kirch Media an den Heinrich-Bauer-Verlag (HBV) und dessen Finanzpartner Hypo-Vereinsbank nichts mehr im Wege zu stehen: Am 30. Oktober spricht sich der Kirch-Gläubigerausschuss für die Annahme des Angebots von Bauer aus, und auch die Kirch-Geschäftsführung lässt verlauten, dass die Entscheidung grundsätzlich gefallen sei. Die Summe für den Verkauf soll bei rund 2 Mrd. Euro liegen, weniger, als sich die Gläubigerbanken erhofft hatten. Der HBV verlegt über 100 Zeitschriften im In- und Ausland und macht damit rund 1,7 Mrd. Euro Umsatz. Unangefochtener Marktführer ist er im Bereich der Programmzeitschriften. Ob sich aus dieser starken Stellung zusammen mit den dann neu erworbenen Fernsehsendern ProSieben, Sat.1, Kabel 1 und N24 eine „vorherrschende Meinungsmacht“ ergeben könnte, solle nach Auffassung der Direktorenkonferenz der Landesmedienanstalten (DLM) medienkonzentrationsrechtlich geprüft werden. Bis Jahresende verzögert sich jedoch der Verkauf an die Bietergruppe um Bauer aufgrund schwieriger Verhandlungen in Bezug auf den Filmrechtehandel. Dies gibt der Bietergruppe um den Amerikaner Haim Saban die Gelegenheit, wieder mit neuen Angeboten einzusteigen, die in den folgenden Monaten ernsthaft in Betracht gezogen werden.

Am 31. Oktober wird die schweizerische Sportrechtegruppe um die Kirch Sport AG (zu der die Bundesliga-Vermarktungs-GmbH und der Fußball-WM-Produktionsdienstleister Host Broadcasting Services gehören) an das eigene Management verkauft, zu dem u. a. der ehemalige Fußball-Nationalspieler und jetzige ARD-Kommentator Günter Netzer gehört. Hauptinvestor sind Robert Louis-Dreyfuß und der Kaffeegrossist Christian Jacobs mit zusammen mehr als der Hälfte der Anteile.

Im Dezember wird berichtet, bei Premiere wolle die Investmentgruppe Permira als neuer Mehrheitseigentümer einsteigen. Hinter der Gruppe stehen $u$. a. verschiedene Banken, darunter die halbstaatliche Bayerische Landesbank, die Bank für Arbeit und Wirtschaft AG sowie die Hypo-Vereinsbank, die die größten Kreditgeber von Premiere sind. Am 3. Dezember wird die Zahl der Abonnenten bei Premiere mit 2,538 Mio. Kunden angegeben. ${ }^{15}$

Ursache für die Insolvenz seines Konzerns war laut Kirch eine Äußerung des Vorstandssprechers der Deutschen Bank, Rolf Breuer, der die Kreditwürdigkeit Kirchs in einem amerikanischen TV-Interview in Frage gestellt hatte. Kirch erhebt daher im Oktober Klage auf Schadensersatz, der Streitwert liegt in Höhe von 100 Mio. Euro. ${ }^{16}$

15 Am 20. Februar 2003 übernimmt die Investorengruppe Permira 65,13 Prozent an der Premiere Fernsehen GmbH \& Co. KG. Weitere Anteilseigner sind die Gläubigerbanken Bayerische Landesbank und Hypo-Vereinsbank mit jeweils 10 Prozent und die österreichische Bank für Arbeit und Wirtschaft, an der die BayernLB beteiligt ist, mit 3,5 Prozent.

16 Das Landgericht München urteilt am 18. Februar 2003, Breuer und die deutsche Bank müssten Kirch alle eingetretenen oder noch entstehenden Schäden ersetzen, da Breuer in dem TV-Interview gegen Verschwiegenheitspflichten verstoßen habe. Der Anwalt Kirchs teilt mit, es dauere noch einige Zeit, bis die Höhe des Schadensersatzes ermittelt sei, eventuell sogar bis zum Abschluss der Insolvenzverfahren in der ehemaligen Kirch-Gruppe. 
Fred Kogel, Programmgeschäftsführer der insolventen Kirch Media, scheidet zum Jahresende aus dem Unternehmen aus, um sich „neuen Aufgaben zuzuwenden“. Seit der Insolvenz von Kirch Media im Frühjahr hatte Kogel vor allem die für das Unternehmen sehr ungünstigen Verträge mit amerikanischen Hollywood-Studios neu verhandelt. Kogel bleibt Kirch Media als Berater verbunden und hält weiterhin 26 Prozent an der von ihm gegründeten Produktionsfirma Kirch Media Entertainment. Kogel war von 1995 bis Ende 2000 Geschäftsführer des zum Kirch-Konzern gehörenden Senders Sat.1. Anfang 2001 wechselte er zu Kirch Media.

Infolge des dramatischen Einbruchs der Werbeeinnahmen gerät 2002 eine ganze Reihe von Ballungsraum- und Regionalfernsehsendern in wirtschaftliche Schwierigkeiten: Am 24. April meldet das zu 100 Prozent im Besitz von Thomas Kirch befindliche TV Berlin Insolvenz an, am 13. Mai folgt das 1996 gegründete Oberfranken TV (die BLM schreibt die Frequenzen neu aus) und am 31. Juli beantragen die drei Gesellschafter des baden-württembergischen B.TV, ein Insolvenzverfahren zu eröffnen. Da zunächst kein neuer Investor gefunden wird, strahlt der Sender ab Anfang Dezember nur noch ein Notprogramm aus. ${ }^{17}$ Beim Kirch-Sender Hamburg 1 übernimmt die Düsseldorfer Deutsche Fernsehnachrichten Agentur am 13. November 92,7 Prozent der Anteile, den Rest behält der Axel-Springer-Verlag. Die im Zuge der Kirch-Pleite von der Medienanstalt Berlin-Brandenburg (MABB) neu ausgeschriebene Frequenz für den Pleite gegangenen privaten Fernsehsender TV Berlin wird am 22. Oktober dem österreichischen Bauunternehmer Soravia erteilt. Die Gruppe hatte bereits die Frequenz des ebenso insolventen TV München übernommen.

Zum 1. Juli wird der private Fernsehsender RTL mit 10 Prozent und 3 Mio. Euro jährlich Gesellschafter der Filmstiftung NRW. Deren Fördervolumen liegt bei ca. 30 Mio. Euro jährlich, die anderen Gesellschafter sind das Land NRW und der WDR mit je 40 Prozent sowie das ZDF mit 10 Prozent.

Die Fachverlagsgruppe Bertelsmann-Springer GmbH übernimmt zum 1. Juli die ausschließlich im Internet erscheinende Netzeitung zu 100 Prozent von der Spray Network N.V. (einer 100-prozentigen Tochter der Lycos Europe N.V.). Der Verkauf erfolgt vorbehaltlich der erforderlichen Genehmigung, insbesondere des Bundeskartellamtes. Die im Herbst 2000 gegründete Netzeitung ist die erste deutsche Tageszeitung, die ausschließlich im Internet erscheint und deren Zugang für alle Interessenten frei ist (www.netzeitung.de). Seit Einführung des Bezahlservices ist es darüber hinaus für Abonnenten möglich, nach individuellen Bedürfnissen Nachrichten aus verschiedenen Themenfeldern, von Wirtschaft über Wissenschaft bis Politik, zu erhalten. Dazu kommen Newsletter und Eilmeldungen per SMS. Mit rund 570.000 Lesern sieht sich die Netzeitung als „eines der populärsten Informationsmedien im deutschen Internet“.

Am 23. Juli wird vor dem Amtsgericht Köln das Insolvenzverfahren zum nordrheinwestfälischen Kabelnetzbetreiber Ish (Calahan) eröffnet. Grund für den Konkurs der Holding war eine Überschuldung, die wiederum durch den gesunkenen Marktwert der Tochter Ish (früher: Kabel NRW) eingetreten sei. Die 4,2 Mio. Kabelkunden würden jedoch weiterhin mit Radio- und Fernsehprogrammen versorgt.

Der neue Vorstandsvorsitzende der Bertelsmann AG ab dem 1. August heißt Gunter Thielen. Sein Vorgänger Thomas Middelhoff, von November 1998 bis Juli 2002 Vorstandsvorsitzender, war zuvor wegen Differenzen mit der Eigentümerfamilie Mohn

17 Nach der Übernahme des Senders durch die Regio Network Communication von Thomas Hornauer am 23. Januar 2003 kann B.TV unter neuer Führung weitersenden. 
zurückgetreten, weil diese seine Pläne zum Börsengang des Unternehmens nicht unterstützte.

Am 2. August einigen sich ARD und ZDF mit dem Rechteinhaber Kirch Media über die Vergabe der Zweitrechte an der Fußball-Bundesligasaison 2002/3. Im Rahmen des Vertragsabschlusses gibt Kirch Media Free-TV- und Pay-TV-Rechte an den Olympischen Spielen 2004, 2006 und 2008 an ARD und ZDF zurück. ARD und ZDF hatten im Januar 2001 beim Kauf der TV-Rechte an der Fußball-Weltmeisterschaft 2002 den Kaufpreis an Kirch Media teilweise mit einzelnen Fernsehrechten an den Olympischen Spielen verrechnet.

Am 3. September geben ARD und ZDF bekannt, dass sie sich mit der Arbeitsgemeinschaft Neuer Deutscher Spielfilmproduzenten e.V. auf eine vertragliche Neuregelung der geförderten Kinoproduktionen geeinigt haben. Danach wird die Rechte-Laufzeit in der so genannten ersten Nutzungsphase von sieben auf fünf Jahren verkürzt, so dass die deutschen Filmproduzenten künftig früher die Rechte an ihren Filmen zur Weiterverwertung zurückerhalten.

Der Axel-Springer-Verlag und der Schweizer Ringier-Konzern verhandeln 2002 intensiv über eine Fusion der beiden Großverlage. Zunächst soll der deutsche Großverlag in einem ersten Schritt den Schweizer Verlag zu 100 Prozent übernehmen, in einem zweiten Schritt soll dann Michael Ringier die Möglichkeit erhalten, Leo Kirchs Springer-Paket zu erwerben und so zweitgrößter Einzelgesellschafter (nach den Springer-Erben) zu werden. Am 14. November jedoch zieht sich Ringier endgültig aus den Gesprächen zurück. Mit der Fusion wäre Europas größtes Pressehaus mit Expansionsmöglichkeiten vor allem auch in Osteuropa entstanden. Springer setzte mit seinen 14.000 Mitarbeitern im Jahr 2001 2,864 Mrd. Euro um und machte 198 Mio. Euro Verlust; Ringier machte im gleichen Zeitraum etwas mehr als eine Mrd. Schweizer Franken Umsatz (rund 708 Mio. Euro), erzielte daraus aber nur einen Gewinn nach Steuern von 34,8 Mio. Schweizer Franken (23,2 Mio. Euro, 3,3\% Umsatzrendite). Das in Zürich ansässige Unternehmen beschäftigt 3900 Mitarbeiter, verlegt in der Schweiz u. a. zwei Boulevardblätter, das Wirtschaftsmagazin Cash, das Nachrichtenmagazin L'Hebdo (Lausanne) und drei Programmzeitschriften, produziert mehrere TV-Sendungen und ist auch in Rumänien, der Slowakei, in Tschechien und Ungarn aktiv (insgesamt 20 Zeitungen und Zeitschriften).

Nach der Zustimmung des Aufsichtsrats der Bavaria Film GmbH am 25. September steht einer Überkreuzbeteiligung der Filmproduktionsfirma mit der Drefa Media Holding (Leipzig), in der die Tochterfirmen des Mitteldeutschen Rundfunks (MDR) zusammengeschlossen sind, nichts mehr im Weg. Das Tauschgeschäft sieht (rückwirkend zum 1. Januar 2001) vor, dass die Drefa Media Holding mit 16,64 Prozent Mitgesellschafterin bei der Bavaria Film GmbH wird; im Gegenzug erwirbt die Bavaria Beteiligungen an insgesamt acht Unternehmen der Drefa. Der Vertrag war bei den unabhängigen Produzenten auf Kritik gestoßen, die bei einer stärkeren Stellung öffentlich-rechtlicher Tochterfirmen Nachteile bei künftigen Auftragsvergaben befürchten.

Vor dem Landgericht München beginnt am 4. November der Prozess gegen die Brüder Thomas und Florian Haffa. ${ }^{18}$ Den ehemaligen Vorstandsmitgliedern der EM.TV \& Merchandising AG wird vorgeworfen, im Laufe des Jahres 2000 die wirtschaftliche Lage des Unternehmens entgegen den Tatsachen als bestens dargestellt zu haben, um so den

18 Die Anklageschrift ist dokumentiert in epd medien 89/2002, S. 25 ff. 
stark gesunkenen Börsenkurs der Medienaktie, die einst ein Star am Neuen Markt war, zu stützen bzw. wieder nach oben zu treiben. Eine solche Handlung gilt als strafbar, wenn dies wider besseres Wissen geschieht und den Börsenkurs messbar beeinflusst.

Die Kommission zur Ermittlung der Konzentration im Medienbereich (KEK) gestattet am 12. November der RTL Group den Einstieg beim privaten Nachrichtensender $n$-tv. Bereits am 5. November hatte die EU-Kommission der Übernahme der Holtzbrink-Anteile (47,33 \%) an n-tv durch die RTL Group zugestimmt.

Am 19. November wird bekannt, dass die defizitäre Online-Buchhandelsplattform BOL, die die Bertelsmann Direct Group zum Ende des Jahres schließen will, vom Online-Buchhändler buch.de übernommen wird. Dadurch wird buch.de nach Amazon der zweitgrößte Online-Buchhändler Deutschlands. buch.de hatte seinen Umsatz im ersten Halbjahr 2002 um knapp 32 Prozent gesteigert. Der operative Verlust sank von 2,2 Mio. Euro im ersten Halbjahr 2001 auf 0,9 Mio. Euro.

Das Bundeskartellamt untersagt dem Holtzbrinck-Konzern am 12. Dezember endgültig die Übernahme des Berliner Verlages, der die Berliner Zeitung, den Berliner Kurier und die Stadtillustrierte Tip herausgibt. Das Kartellamt bekräftigt damit seine vorläufige Entscheidung vom November. Mit Tagesspiegel und Berliner Zeitung würde Holtzbrinck rund 60 Prozent des regionalen Abonnement-Zeitungsmarktes beherrschen. Der nach Holtzbrinck größte Wettbewerber, der Springer-Verlag (Berliner Morgenpost), käme nur auf rund 30 Prozent.

Am 25. November gibt der durch die Anzeigenkrise finanziell schwer angeschlagene Süddeutsche Verlag (SV) bekannt, dass sich die Südwestdeutsche Medien Holding (SWMH) als sechster Gesellschafter am SV beteiligen werde. Die SWMH soll einen Anteil von 18,75 Prozent an der Mediengruppe übernehmen, der Einstieg muss jedoch vom Kartellamt genehmigt werden. ${ }^{19}$ Zuvor war am 31. Oktober der Einstieg der WAZGruppe gescheitert, von der sich der Verlag ein Investment von 100 Mio. Euro erhofft hatte. Der bereits im Sommer gestartete Personalabbau von 650 der 5000 Mitarbeiter soll mit weiteren 300 Stellenstreichungen bis Ende 2004 fortgesetzt werden.

\section{Medienmärkte}

\subsection{Medienangebote / Medieninhalte Presse}

Mit der Ausgabe vom 23. Januar stellt der Verlag Gruner + Jahr sein Wirtschaftsmagazin Bizz ein. Der Zeitschrift hätten im ,anhaltend schwachen“ Vertriebs- und Anzeigenmarkt die „wirtschaftlichen Perspektiven“ gefehlt. Das Blatt, das sich vornehmlich an junge Leute richtete, war im März 1998 als Ableger von Capital gegründet worden.

Die letzte Ausgabe der Wochenzeitung Die Woche erscheint am 8. März. Die Verlagsgruppe Ganske mit Sitz in Hamburg begründet die Einstellung der 1993 gegründeten Wochenzeitung mit „sinkenden Anzeigenerlösen“ und „stagnierender Auflage“. Gespräche über eine mögliche Beteiligung der WAZ-Gruppe waren am 1. März gescheitert.

$\mathrm{Ab}$ Ende Januar ist die komplette Ausgabe der Sächsischen Zeitung als Abrufangebot im Internet verfügbar. Mit einem ISDN-Anschluss dauere es neun Minuten, die etwa

19 Das Bundeskartellamt genehmigt die neue Gesellschafterstruktur am 7. Februar 2003. 
vier Megabyte große Tagesausgabe im PDF-Format zu speichern, teilt die im Großraum Dresden und Ostsachsen vertriebene Zeitung am 31. Januar mit. Das Online-Abonnement kostet monatlich 7,50 Euro, als Zusatzangebot zur Zeitungslieferung 2,50 Euro. Das so genannte „e-Paper“ soll nach Angaben der Chefredaktion vor allem auswärtige Leser erreichen, die mit der Region verbunden sind. 2001 hatte bereits die Koblenzer Rhein-Zeitung ihr Blatt als „weltweit erste Tageszeitung“ komplett ins Internet gestellt.

Die Lage bei der Frankfurter Rundschau (FR) verschärft sich im Laufe des Jahres 2002: Wie andere Zeitungsverlage auch, krankt die FR konjunkturbedingt an einem drastischen Anzeigeneinbruch (- $30 \%$ insgesamt, bei Stellenanzeigen sogar - $50 \%$ ); zusätzlich gingen der hauseigenen Lohndruckerei zahlreiche Herstellungsaufträge anderer Zeitungen verloren. Die Belegschaft der Rundschau (Redaktion, Verlag, Druckhaus) wird bis Jahresende deutlich reduziert.

Das Anzeigenblatt Sunday, das zur Hälfte der FAZ GmbH gehörte, wird am 19. August eingestellt. Grund sei die „wirtschaftlich angespannte Lage“, die „gravierend negative“ Entwicklung des Werbemarkts sowie ein „heftiger“ Medienwettbewerb im RheinMain-Gebiet. Dort war Sunday seit 1997 kostenlos an Haushalte verteilt worden, zuletzt in einer Auflage von wöchentlich 660.000 Exemplaren.

Am 30. August startet das Archiv der Presse über eine neue Internet-Plattform: Unter der Adresse www.archivderpresse.com bietet das Portal die kostenpflichtige Möglichkeit der Recherche und des Abrufs von 30 Mio. Artikeln aus gut 120 Presseorganen aus Deutschland, Österreich und der Schweiz (von Aarganer Zeitung bis Wirtschafts-Woche). Betrieben wird das elektronische Archiv der Presse, das noch weitere Inhalteanbieter gewinnen will, von drei Partnern: vom Dokumentations- und Informations-Zentrum München (DIZ), einem Gemeinschaftsunternehmen der Süddeutschen Zeitung und des Bayerischen Rundfunks; von Genios Wirtschaftsdatenbanken, einer Handelsblatt-Tochter, sowie von APA, einer Tochter der österreichischen Nachrichtenagentur APA.

Trotz der Krise im deutschen Pressemarkt startet Gruner + Jahr im Herbst eine neue 14-tägliche Frauenzeitschrift. Das Magazin Woman soll mit seiner „kompakten und vielfältigen Themenmischung“ sowie einer hohen Nutzwertorientierung „einzigartig auf dem deutschen Markt" sein. Startauflage sind 250.000 Hefte.

Die Süddeutsche Zeitung (SZ) nimmt ab 9. November eine neue Jugendseite ins Blatt. Auf der jeweils am Samstag erscheinenden Seite sollen die besten Beiträge des Projekts „Schule und Zeitung“ erscheinen. Die SZ will ihr Projekt „Schule und Zeitung“ neu organisieren und enger mit dem Online-Angebot www.jetzt.de verzahnen. Sponsor des Projekts ist der Online-Buchhändler Amazon. Die Süddeutsche Zeitung hatte das Projekt „Schule und Zeitung" früher in Zusammenarbeit mit dem Aachener Institut zur Objektivierung von Lern- und Prüfungsverfahren organisiert, nun wird es von den ehemaligen "Jetzt"-Redakteuren betreut. Unter Anleitung der Redakteure schreiben die Schüler häufig in Tagebuchform gehaltene Beiträge, die anschließend in jetzt.de veröffentlicht werden. Damit würden Zeitung und Internet „optimal verbunden“.

Der deutsche Presserat spricht am 11. Dezember drei öffentliche Rügen aus: Die Süddeutsche Zeitung wird gerügt, weil sie einen Leserbrief so gekürzt hatte, dass seine Aussage ins Gegenteil verkehrt wurde. Die Bild-Zeitung wird zweimal aufgrund eines Verstoßes gegen die Persönlichkeitsrechte gerügt. 


\subsection{Medienangebote / Medieninhalte Rundfunk}

Am 7. Januar schicken die Viva Media AG und AOL Time Warner den Musiksender Viva plus auf Sendung. Das Nachfolgeprogramm des defizitären Viva 2 soll ein „CNN des Musik-TV“ mit „weltweitem Korrespondenten-Netz“ werden. Bereits acht Monate später wird das Programmkonzept komplett geändert: Per Telefon und gegen Gebühr sollen die Zuschauer die gewünschten Musikclips auswählen, redaktionelle Inhalte gibt es kaum.

Am 1. März geht der private Auslandsfernsehkanal Channel D mit einem zweiten 24-Stunden-Hörfunkprogramm, dem Channel D Heimatradio, auf Sendung. Zeitgleich mit dem TV-Programm war am 1. September 2001 bereits das Radioprogramm Channel D Schlagerradio gestartet. Die Pay-Programme sind in Nord- und Südamerika im Paket für 20 US-\$ monatlich via Satellit zu empfangen. Am 27. September beantragt Channel $D$ nach rund einjährigem Sendebetrieb beim Amtsgericht Bremerhaven die Eröffnung des Insolvenzverfahrens. Der Sender hatte lediglich 1000 Abonnenten gewinnen können. Dies reichte trotz relativ niedriger Kosten für den Rechteerwerb und den Technikbetrieb nicht aus, um den über einen SES-ASTRA-Satelliten abgewickelten Sendebetrieb aufrechtzuerhalten. Zudem war es infolge der Medienkrise nicht gelungen, neue Investoren zu finden. Die Veranstalter von Channel D, zu dessen Gesellschaftern auch Harald Schmidt gehört, hatten eine Basis von 20.000 Abonnenten angenommen, um bei einem monatlichen Abo-Preis von 20 \$ die Gewinnschwelle zu erreichen.

Am 3. März geht im Bayerischen Fernsehen das Medienmagazin „Einblick“ auf Sendung, am 14. April startet der Norddeutsche Rundfunk um 23.15 Uhr seine neue Medien-Sendung „Zapp“ mit Moderator Gerhard Delling.

German TV, das gemeinsame Auslandsfernsehen von Deutscher Welle (DW), ARD und ZDF, nimmt am 8. April in den USA seinen Sendebetrieb auf. Das 24-Stunden-Programm in deutscher Sprache wird überwiegend aus den Programmbeständen von ARD, ZDF und DW zusammengestellt und bietet größtenteils informations- und kulturorientierte Sendungen. Verbreitet wird German TV über die Globe Cast DTH-World-Television-Plattform via Telstar 1 und GE 1. Globe Cast übernimmt auch die Verwaltung der beim Sendestart rund 800 Abonnenten und den Versand der DVB-Empfangsgeräte. Die Finanzierung von German TV erfolgt mit jährlich 5,1 Mio. Euro aus dem Bundeshaushalt, der Break-Even-Point soll mit 70.000 Abonnenten nach 7 Jahren erreicht werden. Das 15 \$ monatlich kostende German TV kann Ende September 1900 Haushalte als Kunden vorweisen.

Das Kirch-Unternehmen Beta-Research stellt auf der Kabelfernsehmesse Anga Kabel vom 23. bis 25. April ein neues Pay-TV-Verschlüsselungssystem Betacrypt 2 vor. Die zweite Generation des Verschlüsselungssystems war auch nötig geworden, weil Hacker die alte Software verschiedentlich geknackt und die Betriebssicherheit des gesamten Verschlüsselungs- und Abrechnungssystems von Premiere in Frage gestellt hatten

Bei sechs Sendungen der Privatsender SAT.1 („Britt - der Talk um Eins“), RTL („Polizeistation“), Pro Sieben („Bimmel Bingo“ in „TV Total“) und Premiere (zu frühe Ausstrahlung zweier Spielfilme vor 20.00 Uhr) stellt die Gemeinsame Stelle Jugendschutz, Programm, Medienkompetenz und Bürgermedien (GSJP) im März und April Verstöße gegen Jugendschutzbestimmungen und den Verhaltenskodex für tägliche Talkshows fest. Die GSJP kann selbst nicht beanstanden, sondern den beaufsichtigenden Landesmedienanstalten nur Maßnahmen empfehlen.

SAT.1 stellt zum 30. April die terrestrische Verbreitung seines Programms in BadenWürttemberg ein, um damit Verbreitungskosten von mehr als einer halben Million Euro 
jährlich einzusparen. Nur 4,4 Prozent der Zuschauer dieses Bundeslandes empfangen die Fernsehprogramme noch terrestrisch, der Rest über Kabel und Satellit.

Am 15. Mai geht der Reiseverkaufssender Sonnenklar TV mit einem 24-StundenProgramm auf Sendung. Das Programm war zuvor in Fenstern auf 9Live, n-tv, TV München und TV-Berlin gelaufen und wird jetzt als unverschlüsselter Bestandteil des Pay-TV-Senders Premiere und über alle digitalen Free-to-Air-Satelliten-Receiver (Astra $1 \mathrm{H}$ ) ausgestrahlt.

Die Bayerische Landeszentrale für neue Medien (BLM) entscheidet am 14. Juli, dass die Shows „Jackass“ und „Freak Show“ des Musiksenders MTV nur noch nach 22.00 bzw. 23.00 Uhr ausgestrahlt werden dürfen. Die als teilweise schwer jugendgefährdend eingestuften Sendungen, die nach dieser Einstufung nicht (erneut) gezeigt werden dürften, zeigen Stunts und waghalsige „Mutproben“, die zur Nachahmung anreizen könnten. Am 8. Juni hatte sich ein Jugendlicher bei einer Nachstellung eines Stunts aus der „Freak Show“ schwer verletzt. Nachdem MTV zwei Beschlüsse des Bayerischen Verwaltungsgerichthofes gegen den BLM-Beschluss erwirkt, kann MTV die umstrittenen Folgen ab dem 5. November wiederholen.

Am 29. April geht das 24-stündige Unterhaltungsprogramm Tele 5 auf Sendung. Das Programm der Tele München-Gruppe TMG des Filmrechtehändlers Herbert Kloiber bringt hauptsächlich Spielfilme und Serien und ist via Astra $1 \mathrm{~B}$ und im bayerischen Kabelnetz zu empfangen.

Der Privatfernsehsender SAT.1 muss im Magazin „Blitz“ eine Programmrüge ausstrahlen, entscheidet die für die SAT.1 zuständige Landeszentrale für private Rundfunkveranstalter Rheinland-Pfalz (LPR). Anlass für die Beanstandung war die detaillierte Darstellung eines Mordes in der Türkei am 11. Oktober. Das Boulevardmagazin „Blitz“ war bereits mehrfach mit den Programmgrundsätzen und den Jugendschutzvorschriften in Konflikt geraten. Im August hatte sich SAT.1 für die Ausstrahlung eines Amateurvideos entschuldigt, das eine misslungene Rettungsaktion eines Hochwasseropfers zeigte, das in der Elbe starb. Rechtliche Maßnahmen waren damals von der LPR nicht eingeleitet worden.

Das Nachrichtenmagazin Focus erhält am 24. Oktober vom Medienrat der Bayerischen Landeszentrale für neue Medien (BLM) eine UKW-Frequenz für das geplante Focus-Radio, die Veranstalter verzichten jedoch am 29. November, da sie keine Rentabilitätschancen für ein Informationsradio sehen.

Auf der ARD-Hauptversammlung in Köln am 27. November wird beschlossen, dass die ARD-Landesrundfunkanstalten ihre Hörfunk-Fremdsprachenprogramme ab 2003 neu und überwiegend eigenständig organisieren werden, Kooperationen wird es nur in einzelnen Bereichen und zwischen einzelnen Anstalten geben.

Am 2. Dezember öffnet tv.nrw, ein landesweiter NRW-Privatsender mit Stammsitz in Dortmund, sein erstes Lokalfenster. Unter dem Namen köln aktuell bietet es von 17.30 bis 19.30 Uhr aktuelle lokale und regionale Nachrichten und Sportberichterstattung für den Großraum Köln. Produziert wird das Kölner Fenster von der apm medien agentur in Köln, die zu 75 Prozent dem Verlagshaus M. DuMont Schauberg und zu 25 Prozent dem Firmengründer Georg von Auersperg gehört.

Die Deutsche Telekom stellt ihr digitales Pay-TV-Angebot Media Vision zum Jahresende 2002 ein. Das Projekt war im August 1999 gestartet worden und hatte nur rund 60.000 Abonnenten gewinnen können, Gewinne waren damit nicht möglich. 


\subsection{Werbung / Marktanteile}

Das Jahr 2002 ist durch einen drastischen Einbruch der Werbeaufwendungen und Gewinne gekennzeichnet. Der gesamte Werbemarkt verbucht nach Nielsen Media Research ein Minus von 4,4 Prozent.

Die Zeitschriften verlieren mit einem Umsatzrückgang von 7,2 Prozent überdurchschnittlich. Mit einer leichten Steigerung des Brutto-Werbeumsatzes um 1,2 Prozent auf 340,4 Mio. Euro kann lediglich Bauer Media, der Anzeigen- und Onlinevermarkter der Bauer Verlagsgruppe, das Jahr 2002 abschließen. Laut Nielsen Media Research schließt Bauer damit als einziger Großverlag 2002 mit einem positiven Ergebnis ab. Vor allem die Programmzeitschriften TV Hören und Seben und Auf einen Blick trugen mit kräftigen Steigerungen zu dem guten Ergebnis bei.

Ebenfalls ein positives Ergebnis gibt es bei den Online-Bruttowerbeaufwendungen des Jahres 2002: Laut Nielsen Media Research wurden im Internet von Januar bis Dezember 2002 insgesamt 255,9 Mio. Euro für Werbung ausgegeben. Das entspricht einem Plus von 20,9 Prozent zum Vergleichszeitraum des Vorjahres.

Die 15 deutschsprachigen, bundesweit zu empfangenen TV-Sender erwirtschaften nach einer Auswertung von SC Spot-Control brutto insgesamt rund 7,064 Mrd. Euro, das bedeutet im Vergleich zu 2001 ein Minus von etwa 305 Mio. Euro bzw. einen Rückgang um 4,13 Prozent. Die großen TV-Anbieter müssen alle aufgrund der abflauenden Konjunktur Verluste hinnehmen. Marktführer RTL kann zwar mit Brutto-Umsätzen von gut 2,091 Mrd. Euro seine Spitzenposition behaupten, büßt jedoch im Vergleich zum Vorjahreszeitraum knapp 23 Mio. Euro ein (-1,09 \%). Das TV-Unternehmen Pro Sieben Sat.1 Media muss heftige Gewinneinbrüche hinnehmen: Bei einem Umsatzrückgang von 6 Prozent auf 1,895 Mrd. Euro sinkt das Ergebnis vor Steuern um 80 Prozent auf 21 Mio. Euro. Der Jahresüberschuss hält sich dabei im Vergleich zu anderen Medienhäusern mit 15 Mio. Euro zumindest im positiven Bereich. ProSieben hält sein Minus mit etwas mehr als 73 Mio. Euro (4,86 \%) in Grenzen und kommt mit 1,433 Mrd. Euro auf Platz zwei vor dem Schwestersender SAT.1, der 1,404 Mrd. Euro erlöste, 3,14 Prozent weniger. Härter trifft es Kabel 1 mit einem Rückgang um 8,44 Prozent auf 375 Mio. Euro. Den größten Rückschlag verzeichnet jedoch das ZDF, das einen Abschlag von 21,66 Prozent hinnehmen muss und brutto nur noch etwas mehr als 152 Mio. Euro einnimmt. Auch RTL II leidet mit einem Minus von 16,51 Prozent und einem Umsatz von 386 Mio. Euro unter der schwachen Konjunktur. Super RTL erwirtschaftete 159 Mio. Euro (-10,42 \%). Unter den öffentlich-rechtlichen Sendern verbuchte die ARD ein Minus von 16,77 Prozent und einen Umsatz von 176 Mio. Euro. Das Deutsche Sportfernsehen (DSF) hingegen kann seine Brutto-Umsätze um 3,96 Prozent steigern (181 Mio. Euro), die Musiksender Viva (146 Mio. Euro) und MTV (128 Mio. Euro) halten die Vorjahresumsätze bzw. legten gegenüber dem Vorjahr zu. Der Münchner Mitmachsender 9Live, der vor einem Jahr noch TM3 hieß und damals kaum Werbeumsätze machte, verzeichnet ein Plus von 22,08 Prozent und steigerte sich auf 15 Mio. Euro Umsatz.

Mit einem Marktanteil von 14,6 Prozent (alle Zuschauer ab 3 Jahre) bleibt der Privatsender RTL auch 2002 TV-Marktführer, wie bereits in den Vorjahren. In seiner Kernzielgruppe der 14- bis 49-Jährigen kommt RTL sogar auf 17,6 Prozent. Den zweiten Platz belegt das Erste der ARD (14,2 Prozent bei allen ab 3 Jahren, + 0,4 \% gegenüber 2001), den dritten das ZDF, das sich um 0,7 Prozent auf 13,8 Prozent verbesserte. Die Dritten Programme der ARD kommen zusammen auf 13,1 Prozent Marktanteil in der Gesamtseherschaft und damit auf Platz vier. Es folgen Sat.1 mit 9,9 Prozent (0,2 \%), ProSieben mit 7,0 Prozent, Kabel 1 mit 4,5 Prozent und RTL 2 mit 3,8 Prozent. 
VOX kann in der werberelevanten Zielgruppe der 14- bis 49-Jährigen einen Marktanteil von 4,7 Prozent $(+0,4)$ verbuchen, das beste Ergebnis seit Bestehen des Senders. Super RTL kann im Jahr 2002 rund 86 Mio. Euro netto an Werbeeinnahmen erzielen. Großen Anteil am Erfolg des Senders hat die Marke „Toggo“, die das Fernsehen, das Internet (www.toggo.de), Events und Merchandising verbindet. Super RTL hält einen Marktanteil von 18,7 Prozent bei den 3- bis 13-jährigen Zuschauern, vor RTL (12,7 \%), RTL 2 (11,4 \%) und dem öffentlich-rechtlichen Kinderkanal (10,1\%).

2002 müssen die deutschen Zeitungen und Zeitschriften einen merklichen Rückgang der verkauften Gesamtauflagen gegenüber dem Vorjahr hinnehmen. Wie die Informationsgemeinschaft zur Feststellung der Verbreitung von Werbeträgern (IVW) am 22. Juli mitteilt, haben sich die Auflagen der Tages- und Sonntagszeitungen im 2. Quartal 2002 auf das Verkaufsniveau von 1991 verschlechtert. Lediglich die Mediengattung der Kundenzeitschriften habe an Auflage gewinnen können. Verglichen mit dem Vorjahreszeitraum erlitten die Tageszeitungen einschließlich der aktuellen Sonntagsausgaben sowie Sonntagszeitungen erneut einen Rückgang ihrer verkauften Auflage um 600.000 Stück pro Tag und verkauften insgesamt 27,71 Mio. Exemplare. Diese Gesamtzahl setzt sich laut IVW zusammen aus: 17,38 Mio. Abonnements (2/01: 17,63 Mio.), 9,6 Mio. Einzelverkäufen (2/01: 10,08 Mio.) und 0,73 Mio. sonstigen Verkäufen (2/01: 0,61 Mio.). Damit seien die Verkaufsauflagen der deutschen Tageszeitungen „auf das niedrigste Niveau seit dem ersten Halbjahr 1991“ gesunken, so die IVW. Durch die erstmalige IVW-Erfassung der Auflagenzahlen zweier neuer Wochenzeitungen stieg die Verkaufsauflage in diesem Segment von 1,77 Mio. im ersten Vierteljahr 2002 auf 1,87 Mio. Exemplare im aktuellen Berichtszeitraum (jeweils die Ausgaben einer Woche zusammengerechnet). Im Vergleich zum 2. Quartal des Vorjahres, in dem noch 1,91 Mio. Exemplare verkauft wurden, ist jedoch, wie in den übrigen Mediengattungen, ebenfalls ein Rückgang zu verzeichnen. Im 1. Quartal war die Woche (Hamburg) eingestellt worden. Die in der IVWStatistik neu hinzugekommenen Wochenblätter sind die Katholische Sonntagszeitung für Deutschland (vorher bei den Publikumszeitschriften rubriziert) sowie ein russischsprachiges Blatt für hier lebende Russlanddeutsche, die Russkaja Germania. Die Auflagen der Publikumszeitschriften waren sowohl im aktuellen Quartalsvergleich als auch gegenüber dem Vorjahreszeitraum rückläufig. Im 2. Quartal 2002 wurden 122,13 Mio. Exemplare verkauft (2/01: 127,85 Mio.). Diese setzten sich zusammen aus 54,47 Mio. abonnierten Stücken (2/01: 55,18 Mio.), 51,92 Mio. Kioskverkäufen (2/01: 55,89 Mio.), 11,33 Mio. sonstigen Verkäufen (2/01: 12,41 Mio.) und 4,41 Mio. über den Lesezirkel abgesetzte Exemplare (2/01: 4,37 Mio.).

\section{Mediennutzung}

ARD und ZDF fordern in einem Pressekommuniqué vom 18. Februar, eine einheitliche „Währung“ bei der Messung und dem Ausweis der Akzeptanz von Online-Angeboten zu schaffen, bei der die Angebote von „Werbeträgern“ und „Nicht-Werbeträgern“ zusammen beurteilt werden.

Der im August veröffentlichten ARD/ZDF-Online-Studie $2002^{20}$ zufolge ist die Zahl der Internet-Nutzer in Deutschland in den vergangenen fünf Jahren um das Siebenfache

20 Die komplette Online-Studie ist abrufbar unter www.br-online.de/medienforschung sowie unter www.zdf.de. 
gestiegen: 28,3 Mio. Erwachsene ab 14 Jahren und damit 44,1 Prozent der Bevölkerung sind online (1997 waren es erst 6,5 Prozent). Der Anteil der Internetnutzer bei den Männern liegt dabei bei 53 Prozent, bei den Frauen sind es 36 Prozent. Nach wie vor sinkt jedoch der Anteil der Internetnutzer mit zunehmendem Alter: Während in der Altersgruppe der 14- bis 29-Jährigen fast vier Fünftel das Internet nutzen, sind es zwischen 30 bis 39 Jahren zwei Drittel, zwischen 40 und 49 Jahren knapp die Hälfte, zwischen 50 und 59 Jahren gut ein Drittel und bei den über 60-Jährigen lediglich 8 Prozent. Die durchschnittliche Nutzungszeit pro Tag liegt bei 121 Minuten, wobei der durchschnittliche Nutzer 5 Tage die Woche online ist. 67 Prozent der Internet-Nutzer haben schon einmal die Homepage eines Fernsehsenders besucht, 34 Prozent die Website einer Radiostation, gut die Hälfte war auch schon auf den Seiten von Zeitungen und Zeitschriften.

Die Studie kommt außerdem zu dem Schluss, dass das Internet die Menschen nicht dazu verleitet, andere Medien seltener zu nutzen. Es zeige sich vielmehr erneut, dass ein neues Medium alte Medien nicht verdränge, sondern komplementär ergänze: Während die durchschnittliche Internet-Nutzung der Gesamtbevölkerung (inkl. Nichtnutzer) zwischen 2000 und 2002 von 17 auf 35 Minuten gestiegen sei, habe sich auch der tägliche TV-Konsum in dieser Zeit von 203 auf 205 Minuten erhöht. Weiterhin „zurückhaltend genutzt" wird die Möglichkeit, Fernseh- oder Radioprogramme im Internet live oder auf Abruf zu nutzen. So hören nur 160.000 Menschen täglich über das Internet Radio, während täglich 50 Mio. Menschen auf konventionellem Wege Radio hörten. Insgesamt hätten 19 Prozent der Befragten angegeben, schon einmal live Radioprogramme im Internet gehört zu haben. Geringer ist die Zahl der Nutzer, die Live-Videoübertragungen im Internet gesehen haben: Der Anteil liegt bei 12 Prozent und damit zwei Prozentpunkte höher als im Jahr davor.

Der Studie „Jugend, Information, (Multi)Media 2002“ (JIM 2002) des Medienpädagogischen Forschungsverbundes Südwest zufolge gehören Computer und Internet für die Jugendlichen in Deutschland mittlerweile zum Alltag: 93 Prozent aller 12- bis 19Jährigen sitzen mindestens einmal pro Monat in ihrer Freizeit vor dem Computer, im Vorjahr waren es noch 83 Prozent gewesen. Am häufigsten nutzen die Jugendlichen das Internet, spielen Computerspiele, hören Musik oder schreiben. Bei der Internetnutzung liegen die Geschlechter gleichauf. 47 Prozent der Jugendlichen besitzen einen eigenen Computer, 28 Prozent haben Internetzugang. Außerdem besitzen 82 Prozent (2001: $74 \%$ ) ein Handy.

Im Mai 2002 sind einer Studie des Bundesbildungsministeriums ${ }^{21}$ zufolge über 685.000 Computer in bundesdeutschen Schulen im Einsatz, rund 230.000 Geräte mehr als ein Jahr zuvor. Gut die Hälfte der Computer in den knapp 32.500 untersuchten Bildungseinrichtungen sind multimediafähig. In Grundschulen müssen sich danach rein rechnerisch 23 Kinder einen PC teilen, in den Sekundarschulen sind es 17, in den berufsbildenden Schulen 13 Schüler.

Laut Media-Analyse (MA) 2002 für Pressemedien haben die Zeitschriften gegenüber der vorherigen Erhebung rund eine Million Leser hinzugewonnen. Alle Zeitungen und Zeitschriften zusammengenommen hätten rund 265 Mio. Leserkontakte erreicht, teilt

21 Die Studie ist als pdf-File zum Download verfügbar unter www.bmbf.de/pub/it-ausstattung_der_Schulen_2002.pdf. 
die Arbeitsgemeinschaft Media-Analyse (ag.ma) am 24. Juli mit. Dass dennoch weniger Exemplare verkauft worden seien, könnte daran liegen, dass sich immer mehr Haushalte ein Tageszeitungsexemplar teilen, um Geld zu sparen. Obwohl das Angebot auf dem Pressemarkt noch größer geworden ist, haben die etablierten Zeitschriften im Schnitt bei ihrer Leserschaft zugelegt. Von 153 Titeln (Zeitschriften, Wochenzeitungen und Supplements) gewannen 48 an Reichweite, 57 blieben unverändert und nur 48 mussten einen Rückgang hinnehmen. Nicht mehr dabei sind die eingestellten Zeitschriften Die Woche, BIZZ, Online today, Telebörse sowie Motorrad, Reisen + Sport. Zuwächse verbuchten vor allem (wöchentliche und monatliche) Frauenzeitschriften, Lifestyle-Magazine sowie EDV- und Wissenschafts-Zeitschriften, leichte Verluste waren bei der (wöchentlichen und 14-täglichen) Programmpresse, Auto- sowie Wohnzeitschriften und den 14-täglichen Frauenzeitschriften erkennbar. Bei den großen Magazinen hat der Stern seine Spitzenreiterposition nochmals um 140.000 Leser auf 7,91 Mio. Reichweite ausgebaut. Focus stagniert bei 6,18 Mio., wohingegen das Hamburger Nachrichtenmagazin Der Spiegel um 90.000 Leser auf 5,72 Mio. zulegte. Bei den Programmzeitschriften setzte sich Bauers TV Movie mit jetzt 6,88 Mio. Lesern (plus 40.000) an die Spitze. Die Kundenzeitschrift des Pay-TV-Senders Premiere erreicht 2,57 Mio. Leser. Unter den täglichen Kaufzeitungen hat sich Springers Bild um 580.000 auf nun 12,11 Mio. Leser verbessert. Bei den täglichen Abonnementszeitungen konnte sich die Marktführerin Süddeutsche Zeitung (SZ) nochmals leicht um 10.000 Leser auf 1,15 Mio. verbessern. Ebenfalls gewonnen hat Springers Welt, und zwar von 90.000 Lesern auf 610.000 (plus 16 Prozent). Andere große Blätter verloren zum Teil stark: Frankfurter Allgemeine Zeitung minus 40.000 auf nur noch 860.000 Leser, Frankfurter Rundschau minus 20.000 auf 410.000 und das wirtschaftsorientierte Handelsblatt minus 30.000 auf nun noch 510.000 tägliche Leser. Auch die linksalternative taz verlor 10.000 Leser auf 140.000 (minus sieben Prozent). Das Neue Deutschland kommt auf 180.000 Leser. Unter den Wochenzeitungen ist die marktführende Zeit weitgehend stabil: Das Holtzbrinck-Blatt behauptete sich bei 1,03 Mio. Lesern; der leichte Zugewinn betrug 20.000 Leser. Die Welt am Sonntag aus dem Hause Springer lag mit 0,97 Mio. knapp dahinter. Für die neu gegründete Frankfurter Allgemeine Sonntagszeitung, die zuletzt über eine Steigerung ihrer verkauften Auflage auf gut 267.000 Stück (nach IVW) berichtet hatte, liegen noch keine Reichweitenzahlen nach MA-Standard vor. Der Erhebungszeitraum reicht vom 30. September 2001 bis zum 23. Februar 2002.

Die Studie „MiT - Medien im Tagesverlauf“ des Vermarktungsunternehmens IP Deutschland (RTL Group), die im Juli präsentiert wird, ermittelt nach einer Befragung von 1500 Bundesbürgern (ab 14 Jahren) eine Gesamt-Mediennutzungszeit von durchschnittlich insgesamt 260 Minuten pro Tag. Davon würden 150 Minuten ferngesehen, 86 Minuten Radio gehört, 19 Minuten (Tages-)Zeitung und 10 Minuten Zeitschriften gelesen. Das Internet (ohne E-Mails) beanspruche 6 Minuten täglich.

Nach Berechnungen der ARD-Medienforschung betrug die durchschnittliche Fernsehdauer pro Tag 201 Minuten (bei den Zuschauern ab 3). Im Zehn-Jahres-Vergleich (1992) betrug der Zuwachs 43 Minuten (158 Minuten).

Quellen: Funkkorrespondenz, epd medien, DocuWatch Digitales Fernsehen, Presseinformationen der Sender und Landesmedienanstalten, www.spot-control.de, www.wuv.de, www.nielsen-media.de, eigene Recherchen 